\title{
La philosophie comme anthropologie historique
}

\section{Salim Mokaddem}

\section{(2) OpenEdition \\ Journals}

Édition électronique

URL : http://journals.openedition.org/trema/778

DOI : $10.4000 /$ trema.778

ISSN : 2107-0997

Éditeur

Faculté d'Éducation de l'université de Montpellier

\section{Édition imprimée}

Date de publication : 1 octobre 2005

Pagination : 101-111

ISSN : 1167-315X

\section{Référence électronique}

Salim Mokaddem, «La philosophie comme anthropologie historique », Tréma [En ligne], 24 | 2005, mis en ligne le 04 mars 2010, consulté le 19 avril 2019. URL : http://journals.openedition.org/trema/778 ; DOI : $10.4000 /$ trema. 778

Ce document a été généré automatiquement le 19 avril 2019

Trema 


\title{
La philosophie comme anthropologie historique
}

\author{
Salim Mokaddem
}

\section{Les enjeux de la philosophie}

1 Les tentatives et les projets d'instituer des enseignements de philosophie ${ }^{1}$ avant la classe de Terminale se basent sur plusieurs constatations: les difficultés de l'épreuve du Baccalauréat et les échecs patents de nombreux lycéens face aux exigences classiques de la dissertation, du commentaire de texte ; la raréfaction non négligeable des étudiants se destinant aux études philosophiques supérieures (Université principalement); l'intérêt $\mathrm{du}$ public scolaire, extra - scolaire pour les recherches philosophiques, intérêt se manifestant par des tirages importants d'ouvrages de philosophie et une médiatisation de certaines pratiques philosophiques. Le paradoxe étant que les professeurs de philosophie, les professionnels de «la» philosophie ${ }^{2}$ se tiennent à l'écart de cette philosophie aux marges qui pourtant les concerne directement dans la mesure où le professeur de philosophie est le seul garant et d'une discipline et d'un espace sémantique conférant aux philosophèmes leur pertinence et leur vérité. Il est pourtant certain que ces demandes, dont les origines et les motivations restent ${ }^{3}$ à interpréter, révèlent que la philosophie concerne aussi bien les philosophes professionnels (salariés de la fonction publique) que la société civile éclairée ou en quête de sens. Ce qui prouve qu'elle déborde le cadre stricto sensu de l'Ecole et qu'elle engage une communauté politique au sens fort du terme. Notre thèse est qu'elle joue un rôle anthropologique crucial en ce qu'elle est fondamentalement anthropofactrice et garante de l'instance démocratique dans cette anthropogénie constitutive de la philosophie. La philosophie s'alimentant au contact de la non philosophie, il est urgent de savoir en quoi les demandes et les offres de philosophie correspondent à des processus constitutifs des sujets de savoir, et, comment la philosophie se présente comme un processus de subjectivation, de construction de soi, de donation de sens. Si la philosophie est convoquée par ceux qui l'exercent de droit et par une demande sauvage, hors territoire officiel, non négligeable, c'est bien parce qu'elle est 
l'emblème d'une discipline qui n'est pas seulement une discipline scolaire ou académique, comme le révèlent les succès de librairies de P. HADOT, ou de G. DELEUZE, entre autres.

Nous proposons l'hypothèse que la philosophie institue symboliquement et ontologiquement une dimension propre à l'être humain en exposant, disposant, un espace de sens par où il a à s'inscrire pour devenir sujet libre. La philosophie permet aux libertés de s'inscrire dans, par l'historicité dont elle est inséparable pour un sujet en devenir. Donc de produire, à partir de conditions très déterminées après coup, des mouvements et des trajectoires dessinant les figures d'une possible liberté.

\section{Anthropologie philosophique d'un point de vue théorétique}

3 La philosophie est apparue en même temps qu'une forme cognitive de retour sur soi mise en forme par la question platonicienne comme figure gnoséologique des savoirs et en même temps que la démocratie comme structure de délibération collective de l'agora. Démocratie et philosophie sont corrélées: elles surviennent lorsqu'une crise met en demeure l'être humain d'en sortir pour continuer à produire les conditions réelles de son existence. Crise du modèle de souveraineté théocratique et crise des autorités hiératiques et hiérarchiques illustrée par le recours aux sophistes comme alternative aux appropriations politiques des savoirs et des connaissances, avec l'ambiguïé dénoncée par PLATON de confondre le vrai et le vraisemblable ou le moderne et l'authentique (première crise de la post - modernité : l'actuel est - il le réel ?) ${ }^{4}$.

La philosophie naît dans la crise, par la crise; elle est la pensée de sortie de crise qui s'installe en elle pour en sortir ; son symptôme est son remède, son pharmakon ${ }^{5}$. C'est par la philosophie que la conscience du néant advient et c'est aussi par cette conscience critique qui se sait scindée dans son être et son objet que la philosophie se constitue. Ce qui suppose qu'elle est bordée, usinée, travaillée,

doublée, escortée, par un autre qui la stimule, qui la bouleverse, qui l'habite, qui la visite, qui est la cause de son procès de questionnement. La critique philosophique, la crisis de la philosophie, gît en cette capacité d'interroger les bords, les marges de ce qui fonde son corps propre et ses impropriétés de fait ${ }^{6}$. La critique philosophique, la crise de la philosophie - en tant qu'elle est cette crise comme philosophie - devient l'expression du jugement critique comme travail de la crise qui la fait naitre à la fois comme philosophie et comme interprétation du lieu et du moment de sa naissance problématique - trop tôt, trop tard, à terme, prématurée, la venue de la philosophie suppose des cliniques de l'obstétrique et $\mathrm{du}$ météorologique conceptuelles depuis la maïeutique socratique jusqu'aux métaphores hégélienne et nietzschéenne de la chouette de Minerve et du crépuscule de l'actualité intempestive. La philosophie est donc par essence aliénée, altérée, car elle pré - suppose une non - philosophie qui la stimule, la convoque en la provoquant, la fait naître, la porte (autre image de la grossesse), et, en contre - coup, elle reconnait cette non - philosophie en la refusant par un jeu de reflets, d'homologations, de justifications, de coupures épistémologiques, de distinctions (dans les sens les plus divers) qui la consacre par cette procédure de légitimation théorique. On assiste à un double mouvement : la crise sociale, politique, religieuse, fait naître la philosophie qui devient mémoire intériorisée, réception de cette crise. Le philosophe exprime alors l'époque qui lui donne sa singularité : mais il peut aussi bien refouler, masquer, exalter, sublimer, 
déplacer ses origines impures, cette crise qui est cicatrice et cordon ombilical reliant la philosophie à cette non - philosophie qu'elle porte et déporte au risque de s'enliser dans une aporie critique ou dans une auto - interrogation infinie sur son « autonomie » et ses " raisons $d$ être et d'agir ». Car il ne peut pas ne pas habiter, même sous la forme du refus, de l'oubli, du dédain, du scepticisme, du nihilisme, du relativisme, du positivisme, ce qui fonde son être au monde. La crise est au cœur de la prise de conscience de la nécessité de la philosophie comme prise de conscience de cette crise à l'intérieur et à l'extérieur du discours philosophique. La pratique philosophique suppose d'affronter cette marge à l'intérieur et à l'extérieur de la théorisation propre à l'expression philosophique. On peut même dire que cette pratique critique définit la pertinence de l'action philosophique en tant qu'elle la reconnait au travers de sa mise en distance conceptuelle : le concept, en philosophie, a pour but de faire voir, de montrer, d'exhiber le réel de cette crise qui sans cesse la constitue en se prolongeant, et en obligeant le philosophe à inventer, créer, produire, des concepts pour comprendre, rendre conscient, réfléchir, interroger le réel de la crise (comme crise dans et du réel). Savoir suivre les interstices et les lignes de fuite ou de faille - ou de faillite des métastases de la crise dans le champ de la pensée nous paraît depuis longtemps faire l'objet thématisé de la discipline philosophique comme production conceptuelle des champs où ses questions et ses réponses suivent de prés la ligne de fracture qui définit le réel de façon critique.

La conceptualisation a donc un rôle et un sens précis : elle est la science même du réel pour la philosophie au moment où elle rend visible ce qui est invisible à la doxa. Elle permet la formation du jugement (affinement langagier, cohérence formelle, pertinence ontologique, abstraction inductive), elle développe l'efficace critique (mémorisation de l'événement, formalisation cognitive, analyse et synthèse des phénomènes), elle actualise les possibles de l'intelligence (rationalisation des effets, imagination des causes, déduction transcendantale des conséquences). Le rôle anthropofacteur ${ }^{7}$ de la philosophie est indéniable; elle donne à l'être humain une historicité constitutive de son être même sans pourtant s'épuiser dans le moment historique qui la fit apparaître et sans s'inscrire totalement dans le champ de l'historicité pure. Ce caractère historial ${ }^{8}$ transcende l'historicité évènementielle mais s'articule et n'existe que par l'occurrence, l'événement qui le voit naître, qui le fait apparaître. Quand la pensée philosophique apparaît, rien n'est plus comme avant, rien ne le sera jamais plus, car le savoir philosophique porte l'idée de sujet comme vérité de l'être qui se sait pensant: la réflexivité philosophique introduit entre la conscience immédiate et presque virtuelle du monde et l'immuable Réel, un savoir qui met en mouvement et le monde virtuel et les contours du Réel. Ce mouvement, cette intranquillité, cette crise qui stimule la conscience pour la porter là où elle n'est pas, et lui faire quitter définitivement le foyer insouciant de l'Etre, c'est ce qui promeut l'histoire dans sa dimension de sens, de changement, de récit, de signification. C'est parce que la non - philosophie, la crise, l'inconfort, oblige la pensée à devenir philosophique (philosophante), que l'histoire devient le lieu de réalisation ontologique du sens, des discours, des actes. Le savoir de la philosophie produit par la crise, institue un sujet du savoir et un sujet d'action. En un sens strict, sans philosophie, l'accomplissement de l'homme comme sujet libre serait improbable 9 . Toute anthropologie est par essence philosophique. C'est aussi pour cela que toute mort de la philosophie (c'est - à - dire toute réduction de la philosophie à une anthropologie, pragmatique ou non) est une mort de $l^{\prime}$ homme ${ }^{10}$. La dimension symbolique de la philosophie comme exercice de soi dans le jeu des vérités, dans un cadre critique permettant de rendre visible les bords et les marges qui la constituent continûment, est ce qui garantit un mouvement de réalisation de soi, 
ou de subjectivation déterminée. C'est peu dire que l'enjeu de la philosophie s'avère plus que vital.

6 Les structures de la connaissance philosophique permettent donc d'inscrire une conscience réflexive, une conscience critique, une conscience épistémologique qui dessine un espace par lequel le sujet advient en se construisant: il n'est que cette interaction historique, phénoménologique, instituant un devenir soi à partir de l'autre (de ce bord qui le définit négativement et par lequel il se pose en s'op - posant). La philosophie pose la nécessité d'un devenir sujet; elle rend la crise visible par une sorte d'auto - réversibilité de l'autre et du soi. C'est pour cela qu'il est important que la philosophie soit enseignée partout, tout le temps, de façon à déterminer les marges qui fondent, déterminent, structurent les savoirs et les conditions des savoirs. La fonction cognitive de l'activité philosophique éveille l'humanité à elle - même et lui ouvre un espace de subjectivation sans lequel rien n'a de sens ni de valeur. Sans la philosophie, la vie redevient simple vie animale, biologique, _-- (zoé) et non plus _-_-- (bios) ${ }^{11}$. Une vie philosophique ${ }^{12}$, c'est d'abord une vie par la philosophie qu'elle indique : c'est - à - dire, la capacité de voir ce qui ne se voit pas, et de rendre invisible ce qui se voit trop, par un jeu d'analyses des séries de sens et de possibles qui composent notre horizon de "vérité ». Chaque savoir, chaque vérité, repose sur un ordre qu'il faut comprendre, dont il faut se déprendre, pour ne pas être agi par la structure qui le commande. Etre sujet, c'est refuser l'assujettissement qui nous fait sujet pour un donné non interrogé. C'est ensuite prendre position par des mouvements dans ces séries constituantes afin d'en explorer les limites, les impasses, les chemins inconnus. Penser philosophiquement consiste donc inévitablement à prendre des risques. D'abord celui de se perdre dans la pensée; contrairement à ce que pensait KANT ${ }^{13}$, à son époque, l'aujourd'hui réclame qu'on puisse se déprendre de soi, afin de résister à la doxa à l'ère de sa reproductibilité technique ${ }^{14}$. Penser le présent, le saisir dans le " point nocturne de la contradiction ", selon l'expression hégélienne, c'est - à - dire là où le réel perd sa raison d'être et exprime la dissolution du sens, c'est cela le travail philosophique ${ }^{15}$.

Il y a une pédagogie double de la philosophie : elle donne à connaître, à savoir par le biais des concepts et des systèmes rationnels qu'elle produit en son histoire, comme discipline, et, elle inscrit sur les bords internes le rappel historial, critique, des conditions de son émergence, de sa fragile raison d'être. En ce sens, elle participe de la science du point de vue de ses règles, de ses objets, de ses méthodes, de ses processus de formalisation, de ses noms de véridicité. Mais elle est plus fondamentalement liée à un processus de subjectivation où il y va de la création autonome, plastique, d'un sujet par la façon dont il s'inscrit dans la tradition et l'hyper - modernité philosophique. La question de la philosophie passe par celle d'une redéfinition du nœud qui le soutient au réel de son apparition et de sa fragile institution. Le sujet philosophique est celui qui distingue l'histoire philosophique de l'histoire de la philosophie (sujette à interprétation, à l'instar de la nature pour les physiciens, ou du fait psychique pour les psychologues...).

\section{Les objets de la philosophie}

La philosophie ne peut être générale, ou supra - historique ; elle ne peut d'ailleurs être la philosophie car elle est singulière, expression d'une multitude de complexités dialectiques mêlant l'historicité des pratiques théoriques et l'évènementiel de leur situation. Par contre l'activité philosophique se caractérise par un certain nombre de 
démarche logiques et politiques, au sens où la crise, le conflit, la fracture se traduisent socialement et que la réflexion critique - la reprise sous la forme d'une conceptualisation critique - se doit de refléter l'événement en tant que tel. D'où le caractère non pas tant inachevé de la praxis philosophique que processuel; ce qui est difficile est d'articuler l'engendrement, le commencement, en philosophie, avec le résultat qui est le tout social dans son apparence immédiate. Justement parce qu'il n'y a pas de philosophie doctrinale, de système vrai, de dogme, il ne peut être question de recourir à des essences éternelles, des faits naturels, ou des éléments égologiques : rien ne pré - existe épistémologiquement à la rationalité philosophique parce que tout lui pré - existe socialement, ne serait - ce que sous la forme du langage de l'évidence, ou de la découpe entre travailleurs manuel et intellectuel.

9 Ainsi, on ne peut réduire l'activité philosophique à une idéologie que si elle ne réfléchit pas les conditions de sa production; si elle considère que l'aliénation qu'elle porte, qu'elle est en quelque façon, est indifférente, elle risque de devenir une morale de l'opinion, une science du général, ou un discours occultant les contradictions réelles d'où elle tire ses forces et ses faiblesses. Penser suppose donc une schize entre ce qu'on est, ce qu'on pense de ce qu'on est, et ce qu'on est en réalité quand on se déprend - quand on se dé - pense de " soi », de ce soi construit, produit, assujetti à une forme, à un état, à une situation liée à un complexe historique dont il faut débrouiller l'écheveau.

10 Est - ce à dire que tous les objets de la réalité, tous les thèmes, tous les évènements peuvent faire l'objet d'un travail philosophique? On se souvient du dédain de Platon pour les objets infâmes (la boue, la crasse, le sensible...), comme d'un ton grand seigneur qui ignore les «basses réalités» de ce monde. La philosophie est engagement: tout la convoque, la concerne ${ }^{16}$.

11 Tout la convoque parce que tout est agité, porteur, de cette crise qu'elle prolonge, qu'elle assume. Dont elle est l'assurance de sa mémoire, même dans les formes caractéristiques de l'« oubli » qui la contraint à ne pas oublier cet oubli : on peut interpréter une certaine philosophie contemporaine, très morale, très post - moderne, comme une tentative d'esquiver l'essence de cette crise par le recours à des vertus petites ou à des idées générales sur les civilisations, le but étant d'empêcher que la pensée soit autre chose que la forme subjective de l'interprétation idéaliste du réel. Nous n'avons pas le temps de développer ici, mais disons que la vente des titres de philosophes très médiatisés - ce que BOURDIEU appelle avec justesse des "philosophes de télévision» - s'explique par la violence répétitives des communications publicitaires basées sur la répétition, le visuel accrocheur, la simplicité des messages, et l'univocité des références, et, aussi et surtout, par la réduction de la philosophie à une certaine consultation psychosociale de type religieux. Le philosophe - gourou, donneur de conseils, sage, savant, ayant remplacé ici le traditionnel clerc, confesseur et censeur.

L'activité pratique de la philosophie s'arc - boute sur une conscience aiguë des contradictions du réel, sur la constatation d'une crise, d'un malaise, d'un désordre d'une ligne sensible, nécessitant une clarification, une orientation, une carte de lecture ${ }^{17}$. Les objets de la philosophie sont ceux que le réel lui porte quand elle se sait prise en lui, exprimée par lui, structurée par lui. Il faut bien donner à la philosophie une fonction si l'on ne veut pas en faire une activité culturelle indifférente aux déterminismes divers du 
monde. Si la philosophie mérite qu'on reste plus qu'une heure dans son cabinet de travail, c'est qu'elle oblige à une révolution radicale: vaut - il la peine de penser la vie, les valeurs, le sens, la vérité si on ne peut les connaître que par le concept (réduit à une simple catégorie de l'entendement)?

C'est la question que pose fondamentalement la doxa à la philosophie et que la philosophie reprend à son compte : à quoi sert de philosopher? faut - il apprendre à philosopher, la philosophie? Qu'est - ce qu'être philosophe aujourd'hui? Que nous enseigne de nécessaire à nos libertés l'expérience de la philosophie ?

Justement cela : l'expérience n'a de sens qu'à être préservée de sa disparition par le fait de la disposer à l'ouverture de son jeu et de ses virtualités réelles ${ }^{18}$. La philosophie préserve ontologiquement l'expérience comme qualité vivante, anthropofacturale; et, elle assure, de ce fait, l'historicité constitutive d'une marche pour (par) l'homme. Le travail de la philosophie se déroule en elle pour l'autre qui la borde ; la non - philosophie est l'espace par lequel les lignes de tension et de fracture vérifient les processus des concepts philosophiques.

\section{Ouverture}

Les enjeux de la philosophie sont multiples du fait de cette crise qui la constitue, la fonde, la suture au risque de la voir s'auto - occlure dans le réel et de s'exclure de la ligne de partage la définissant. Au vrai, il est impossible d'échapper à l'inscription nécessaire gisant au cœur de ses problématisations et des styles qu'elle donne à celles - ci : l'impossible de cette échappée est son sérieux même et le tragique nécessaire de l'urgence qui l'habite. Le philosophe sait qu'il est contraint par la façon dont il s'installe dans sa praxis théorique. Les enjeux de son discours demeurent :

- historiques, parce que la philosophie a pour tâche d'être la mémoire historiale d'une crise politique et indissociablement métaphysique définissant le sens de son activité et de ses pratiques de vérité

- épistémologiques, car sans la philosophie (ou les philosophies) aucune discipline ne peut délimiter le degré de réflexivité, de retour sur soi, nécessaire à la constitution de son champ et à la pertinence de ses objets, problématiques, limites, territoires, formes de véridicité internes

- anthropologiques, puisque la philosophie, travail du concept, institue l'humanisation comme horizon de sens pour nos libertés, et puisqu'elle engage enfin à se situer sur une ligne, un bord, une fracture par laquelle la praxis philosophique autorise une sortie du non oubli, de l'occultation, du refoulement, de la distanciation qui oblitère les urgences des réactions les plus quotidiennes contre ce qui ramène nos vies à un en - deçà de la démocratie questionnée et inventée par la philosophie ${ }^{19}$.

Pour toutes ces raisons, la philosophie devient plus nécessaire que jamais. Sans elle le présent devient plus obscur, moins présentifié. L'avenir s'estomperait dans l'incertain brumeux de la fausse répétition, et, surtout, tout processus visant à objectiver et subjectiver ce qui fait l'épaisseur opaque du monde se dissiperait dans les avenues encombrées de l'idéologie post - moderniste et des régressions dogmatico - sceptiques facilitant les asservissements aux maîtrises du jour. Nous méritons mieux que les idoles 
de la communication et les fausses vérités éclatantes des fins de partie où la violence devient la positivité de la globalisation des savoirs et des connaissances quand ils forclosent leur point névralgique de constitution. Cela suppose que le courage de penser passe aussi par celui de l'énonciation: les énoncés philosophiques se reconnaissent à ce style et ce tremblé qu'ils imposent aux paresseuses "interprétations » de la réalité confondues avec les formes de l'ignorance véhiculée par les savoirs modernes de la résignation.

\section{BIBLIOGRAPHIE}

PLATON, Cuvres complètes, trad. ROBIN, 2 volumes. [Paris] : Pléiade.

CHATELET, F., PLATON, Idées. Paris : Gallimard, 1965.

KANT, E., Cuvres philosophiques, 3 volumes. [Paris] : Pléiade, 1980, 1985, 1986.

HEGEL, G.W.F., La phénoménologie de l'esprit, trad. J. HYPPOLITE, 2 tomes. Aubier, 1941 ; Principes de la philosophie du droit, trad. R. DERATHE. Paris : Vrin, 1982.

MOKADDEM, S., FOUCAULT. Une vie philosophique. Montpellier : 2000 ; De la vie à la mort. Un procès sans sujet, in Phénoménologie et psychanalyse, dir. J. - C. BEAUNE. Champ Vallon, 1998.

FOUCAULT, M., Dits et écrits, 4 volumes. Paris : Gallimard, 1994.

DELEUZE, G., L'île déserte et autres textes. Paris : Minuit, 2002 ; Mille plateaux (avec F. GUATTARI).

Paris : Minuit, 1980.

AGAMBEN, G., Moyens sans fins. Paris : Payot, « Rivages ».

BENJAMIN, Walter, CEuvres, 3 volumes. Paris : Folio, Gallimard, 2000.

BALIBAR, E., Droit de cité. Paris : Editions de l'Aube, 1998.

\section{NOTES}

1. Dans les Ecoles, collèges, lycées hors classe terminale, les lycées professionnels, les Facultés de médecine, les Ecoles d'ingénieurs, d'Arts (appliqués ou non), etc., des enseignements très hétérogènes de philosophie sont çà et là dispensés. Les Etats généraux de la philosophie, tenus en Sorbonne en avril 2003, très décevants quant aux analyses des situations complexes qui font le quotidien des praticiens de la philosophie, n'ont aucunement été à la hauteur de ce qui secoue actuellement l'enseignement de la discipline. Pour faire court, l'enseignement de la philosophie recherche encore sa technè, son art et sa manière propre. Pour l'heure, seuls les concours et les procédures académiques de formation des enseignants, définissent les profils des acteurs de la philosophie avec le succès et les impasses dus aux inerties historiques déjà relevées par Ferrari. $\mathrm{A}$ côté de cela, des intronisations sauvages de "philosophes » s'autorisant d'eux - mêmes distillent l'idée que la philosophie est une activité spontanée, ne nécessitant qu'une « attitude », voire une "disposition " réflexive immédiate, sans origine ni fondement autre que la simple "décision ». Cette culture de la philosophie "originaire», libérée - pense - t - elle - des contraintes 
historiques, scientifiques, de la rationalité des procédures philosophiques, débouche sur une barbarie sans précèdent: la philosophie serait réduite à une misère auto - instituante de l'appropriation illocutionnaire. Le premier qui dit : «Je suis philosophe » le serait par les voix de l'Esprit saint lui conférant la grâce du génie philosophique et le droit -confondu avec l'usurpation factuelle de titre - à l'exercice du délire. Outre le côté comique de ces déclarations sans légitimité autre que celle d'un narcissisme déclamatoire, ce sont souvent les publics (scolaires ou non, majeurs ou non) qui font les frais de l'incurie en étant floués sur ses libidines sciendi. Du coup, la misologie anti - philosophique s'alimente aux prétentions barbares véhiculant au nom de la philosophie tout ce à quoi les philosophes se sont toujours opposés : doxa, lieux communs, dogmes, débats éristiques, sophismes fallacieux... Le logos se transforme alors en rituel d'énonciation sans enjeux définis.

2. L'enseignement philosophique est - il l'enseignement de la philosophie? L'enseignement par des philosophes de philosophèmes particuliers? L'enseignement de professeurs de philosophies et de philosophes choisis institutionnellement par le Conseil National des Programmes? Ou encore l'enseignement de professeurs de philosophie visant à définir, normer, s'approprier la "philosophicité » de leur philosophie professorale? Il y a bien des questions sur la philosophie des professeurs et sur les rôles joués par les garants des institutions philosophiques. Une étude philosophique et sociologique des fonctions d'Inspecteur général, d'Inspecteur pédagogique régional, de conseiller pédagogique, de Président de jury de concours, serait édifiante pour comprendre la formation des enseignants de philosophie, l'« esprit » d'un corps assez retiré des enjeux philosophiques des demandes de philosophie émanées de la société civile, et, par extension, de la cooptation inique, non fondée en raison, régnant de façon scandaleuse dans les Départements de philosophie des Facultés, où les « spinozistes » normaliens ou affiliés s'opposent aux «husserliens » normaliens, entre autres batailles de billevesées valant bien celles qu'on dénonce sous couvert de modernité scientifique ...

3. Pour une interprétation de ces silences institutionnels, de la difficulté des élèves à travailler la philosophie scolaire, des représentations du travail conceptuel par les élèves des classes terminales pendant leur scolarité et après celle - ci, voire les travaux du GER sur les évaluations disciplinaires dirigés par M. FRATISSIER, formateur à l'IUFM de Montpellier, et notre étude sur «La philosophie des élèves » (IUFM de Montpellier, 2001) sont utiles à la compréhension de ces problèmes.

4. On lira avec profit le livre pédagogique de F. CHATELET, PLATON, Idées / Gallimard, et Le sophiste de PLATON, pour la définition et les enjeux de la sophistique. L'écroulement des modèles conservateurs et l'effondrement des repères traditionnels, traditionalistes, de la pensée de Platon peuvent induire que la « révolution » de PLATON dont parle HEGEL dans la Préface aux Principes de la philosophie du droit de 1821 est aussi un besoin d'ordre, de retour à une stabilité exprimés par une crise de l'univers aristocratique athénien du Ve siècle avant J.C. Est - ce à dire que la philosophie historiquement porte en elle cette tension dialectique entre une révolution totale qui change l'être même du monde et un retour à l'ordre ancien d'où elle provient au moment même où elle se constitue dans les formes où nous la reconnaissons aujourd'hui ? On peut, en effet, concevoir qu'il existe une aspiration réactionnaire et une tendance révolutionnaire dans chaque philosophie. Comme exercice, on peut s'amuser à reconnaitre par exemple un HEGEL conservateur - dit, trop rapidement au vu du contexte historico - politique dans lequel il écrit, de « droite »- et un Hegel de " gauche » (pré - marxien, duquel il faudrait faire la coupure pour être à la "gauche de la gauche»), un DESCARTES augustinien ou thomiste, et un DESCARTES copernicien ou galiléen, un SPINOZA plotinien et un SPINOZA cartésien, un MARX « hégélien » et un MARX «marxiste "...Cette découpe se retrouve dans ce qu'Althusser a appelé «la lutte de classe dans la théorie " dans, e.g., LENINE et la philosophie, et même à l'intérieur d'un même corpus, d'une même idée. La coupure entre 1845 et après l'Idéologie allemande ferait un MARX pré - marxiste et un MARX encore "philosophe spéculatif "; cela se retrouve dans Positions 
d'ALTHUSSER où le normalien concède, après lecture de LACAN, que l'idéologie, l'imaginaire, ce qui constitue la vérité $\mathrm{du}$ « sujet » comme fiction du savoir est indissoluble dans la science. Mais cette position elle - même peut faire l'objet d'une dialectisation surdéterminée : pour qui y aura $\mathrm{t}$ - il toujours de l'idéologie ? Et d'où se place le mathème lacanien du $\mathrm{R}-\mathrm{S}$ - I pour penser l'action de l'analyste sinon dans une fiction du one man'show se faisant lui - même ? Pour tout ceci, on lira avec profit R. CASTEL, Le psychanalysme, Maspéro, Paris et le bel article de P. BOURDIEU sur l'identité et ses affres contradictoires , "L'identité et la représentation" (in Actes de la recherche en sciences sociales, $n^{\circ} 35$, novembre 1980, pp. 67 - 72). Pour la question de la périodisation et des versants politiques des enjeux de la théorie chez MARX, le livre d'Etienne BALIBAR, La philosophie de MARX, La Découverte, apparaît plus que pertinent dans ses conclusions.

5. Au sens donné par J. DERRIDA, in "La pharmacie de PLATON» in L'écriture et la différence, Seuil, Points.

6. L'insolence du philosophe vient de ce statut « dérangé » qu'il occupe de droit et de ses vertus à être son époque en tant qu'il met en forme conceptuelle le temps qui est le sien propre ; ce qui se traduit dans son angoisse, son inquiétude, son style, son inédite labilité plastique. L'art est philosophie quant à son contenu et quant à ses soucis pédagogiques de manifestation. Nous reviendrons dans un travail prochain sur ce qu'est la philosophie dans le temps qui la promeut.

7. Les travaux de Peter SLODERJIK sur l'anthropogénèse et l'anthropofacture des processus culturels, sociaux, conceptuels, dans le cadre de la domestication du genre humain, ainsi que les travaux de M. FOUCAULT sur le rôle des disciplines et des noms, révèlent à quel point les idées ne sont jamais idéalistes que pour ceux qui les confondent avec des représentations ou des reflets des choses.

8. Henry CORBIN, traducteur de Heidegger, premières sections de Sein und Zeit (1927), rend la dimension transhistorique et infrahistorique de l'être humain, par ce terme emprunté à la philosophie médiévale (cf. Jean DE BEAUVAIS, Imago Mundi). Nous l'employons dans un tout autre sens que Corbin qui en fait une dimension spirituelle de l'âme, et en un sens propre de ce que l'on pourrait appeler un transcendantal empirique, si les termes pouvaient dépasser leur opposition pour signifier le concept au sens très hégélien du terme : «Le concept est le temps qui est là »«Der Begriff ist die Zeit der Da ist » (Phénoménologie de l'Esprit, Préface).

9. Tout l'enjeu de la philosophie depuis PLATON, DESCARTES, KANT, HEGEL, est résumé par cette proposition hégélienne : «La substance vérité est sujet ».

10. Ce leit - motiv nietzschéen, repris par M. FOUCAULT à la fin de Les mots et les choses (1966, Gallimard), signifie que l'histoire peut faire oublier le processus par lequel s'institue la subjectivité. Le thème post - moderniste de l'immanence n'est en fait qu'une anthropologisation des intuitions du NIETZSCHE des dernières années: l'absence de transcendance implique la transcendance de l'absence. D'où les fins de l'histoire, de la métaphysique, de l'universel, etc. Nous remarquons que le post - modernisme est la traduction en termes philosophiques du refus de prendre en compte à l'intérieur des raisons philosophiques la crise qui fonde les énoncés philosophiques. Le postmodernisme vise à détruire la dimension historiale de la philosophie; en un sens, il est donc régression anthropologique à l'avant - philosophie, à l'avant - démocratie. Nous osons dire qu'il est l'idéologie politique préparant la destruction de l'histoire comme science du réel, et de la démocratie comme figure historiale de la liberté.

11. Pour cette distinction, on lira Giorgio AGAMBEN, Moyens sans fins, Rivages, Payot.

12. Nous caractérisons ainsi l'activité intellectuelle aujourd'hui : un diagnostic du présent à partir d'un savoir des pratiques discursives qui énoncent les problèmes du moment, les formes et les sujets des vérités qui constituent les possibles de l'époque. Cf. Salim MOKADDEM, FOUCAULT. Une vie philosophique, Montpellier, 2000 (dernier chapitre précisément).

13. Dans "Qu'est - ce que s'orienter dans la pensée ?", Kant appliquait le criticisme à la totalité de l'expérience, fût - elle expérience de soi. La pesée kantienne est une pensée de la finitude qui considère le monde comme une position d'un sujet le constituant au moment où le recevant, il le 
connaît à l'aide de catégories et de principes transcendantaux. Nous ne croyons pas en un transcendantal qui précède l'expérience, ni en une expérience qui n'aurait pas la grammaire de son ordre, sa logique au sens du Hegel de la Science de la Logique (1812 - 1816).

14. Il serait intéressant et spirituel de reprendre l'article de W. BENJAMIN en changeant le mot « art » et en lui substituant le mot « information ». On pourrait peut - être comprendre ce qui fait l'aliénation "savante " des masses qui en savent long sur la "jet set», et très peu sur les conditions de sa production sociale, à l'inverse du XIXe siècle où, BALZAC mis à part, les littératures occultaient les processus d'accumulation propre à la bourgeoisie.

15. Après Auschwitz, après la guerre de colonisation mondiale déclenchée par les USA contre tout ce qui nie les intérêts du dollar, cette proposition prend un sens inédit. Formulée pendant les guerres européennes napoléoniennes, elle signifie que l'activité du concept n'est pas le calme olympien de la contemplation joyeuse spinoziste, mais l'installation délibérée dans la part maudite au cœur de la raison. Le silence des "philosophes » français sur l'œuvre de BATAILLE (hormis FOUCAULT, DELEUZE), malgré les emprunts - LACAN prend le concept d'Impossible (d'acéphale) et DERRIDA celui de la déconstruction (a - théologie) à l'auteur de la Somme a théologique - signifie qu'il est difficile de travailler en servant les maître du jour.

16. C'est le mérite de DELEUZE d'avoir rappelé les importances des objets mineurs, et des errances thématiques en philosophie ( $\mathrm{Cf}$. Mille plateaux, Minuit). Les étudiants studieux qui font carrière en philosophie à partie de thèses misérables sur SPINOZA, KANT, PLATON, DESCARTES, etc., appauvrissent la ligne sensible de ces auteurs plus qu'ils ne la suscitent. Nous mettons au défit quiconque aime produire dans la philosophie de trouver une seule thèse depuis ces dix dernières années, par exemple, sur SPINOZA ou HUSSERL, qui ne soit pas le rappel reformulé des thèses de GUEROULT, HEIDEGGER, MATHERON ou RICCEUR. Ce qui est à déplorer n'est pas l'appauvrissement de l'imagination philosophique, mais la standardisation des directions d'études. Le danger pour la philosophie vient des mandarins, chiens de garde, professionnels des jurys de censure et des commissions de recrutement qui savent, d'instinct, orienter les pensées et sélectionner leurs «disciples». La philosophie, en France, est devenue une annexe des programmes de philosophie pour futurs agrégés. Ce qui explique le désert universitaire et l'ambiance de coupeurs de tête qui y règne. Nous sommes en tout point d'accord avec les conclusions tardives, très fin de carrière, de Jean - Claude MILNER, dans Y a $t$ - il encore une vie intellectuelle en France?, Verdier, 2002. La chance de la philosophie, aujourd'hui, c'est son absence au sein de l'institution universitaire: les étudiants et intellectuels sont légions qui vont vers d'autres Départements, moins agités par le ressentiment et la haine de la recherche libérée des pesanteurs de la reproduction des petits maitres. Si nous laissons la question politique de l'autonomie de l'Université, on peut se demander comment se recrutent les élites en France et, surtout, comment sont - elles formées (pour être à même de gouverner, quels sont les critères de compétence requis?).

17. Voir Salim MOKADDEM, «FREUD et HUSSERL : De la crise au Malaise », dans Phénoménologie et psychanalyse, dir. J - C. BEAUNE, éd. Champ Vallon, 1998. Qu'est une philosophie qui ne s'ancre pas dans ce qui convoque la raison à penser son autre sinon une idéologie de la totalité achevée ? Il ne faudrait pas confondre le philosophe avec PANGLOSS, Maître JACQUES, ou le travail intellectuel avec la prose des nouveaux « nouveaux » philosophes.

18. Giorgio AGAMBEN a très vite vu que la "destruction de la raison» (ADORNO en a conceptualisé le sens dans la dialectique négative dans Minima Moralia, Payot) s'accompagne toujours d'une destruction de l'expérience de vie (bios) pour l'humain réduit à la vie animale (zoé) par l'abolition de la mémoire, du jugement réfléchissant, de la formalisation. Nous dirons cela de façon plus hégélienne : on ne peut rien savoir sans l'expérience de l'expérience qu' est la conscience philosophique quand elle dépasse la conscience immédiate de la vie et qui fait l'inconscient historique d'où elle tire sa réalité et sa vérité. Penser philosophiquement veut dire : 
penser la réalité sociale, politique, historique («idéologique») qui détermine l'essence de l'homme.

19. Démocratie qui ne signifie certes pas cette forme inédite de domination bio - politique produite par les sociétés de contrôle occidentales analysée avec précision par Michel FOUCAULT.

\section{RÉSUMÉS}

La philosophie est une pratique conceptuelle qui déborde le cadre disciplinaire de l'enseignement en ce qu'elle institue un rappel historial des conditions anthropofacturales de la démocratie et des savoirs.

Philosophy seems to be an actual and vivid activity not only inside prestigious and academic institutions but also outside schools, colleges and universities.

Actually, the revival of conceptual discussion and public dialogues, hold on its anthropological features. Philosophy and democracy are similar in origins and aims; studying their values and rational occurrences, which reveal the importance of «logos» for solving crisis and conflicts in all societies based on discourses. It's impossible to not link our way of thinking, knowing, learning, criticizing without this research of truth and freedom determining the presence of the particular process which gives to philosophical activity its contemporary acute, sharp, useful, modernity.

\section{INDEX}

Mots-clés : philosophie, démocratie, liberté, pouvoir, modernité, ligne, fracture, crise, historial, production de concept

Keywords : philosophy, knowledge, contemporary crisis, anthropological presence, criticism, humanism - humanity, truth

\section{AUTEUR}

\section{SALIM MOKADDEM}

Maître de conférences en philosophie, IUFM Montpellier 